\title{
Evolución de la distribución de tensiones en un cuerpo cerámico durante la cocción. Parte 1: Planteamiento de los problemas
}

\author{
H. CAMACHO ${ }^{1,2}$, M. E. FUENTES ${ }^{3}$, L. FUENTES ${ }^{1}$, A. GARCÍA ${ }^{1}$, A. PÉREZ ${ }^{1}$ \\ ${ }^{1}$ Centro de Investigación en Materiales Avanzados. Chihuahua, Chih. 31109 México \\ ${ }^{2}$ Universidad Autónoma de Ciudad Juárez. Instituto de Ingeniería y Tecnología. Ciudad Juárez, Chih. 32310 México \\ ${ }^{3}$ Universidad Autónoma de Chihuahua. Facultad de Química. Ciudad Universitaria. Chihuahua Chih. México
}

\begin{abstract}
El estudio de las tensiones durante la cocción de piezas cerámicas es el principal objetivo de este trabajo. Para distintas condiciones de cocido que incluyen el prensado en caliente y la cocción tradicional se plantea un problema de equilibrio de tensiones. Además, se tienen en cuenta varios comportamientos constitutivos que incluyen el Modelo de Riedel para policristales, el Modelo de Celdas de Scherer para amorfos y el modelo de Kahn para fluencia según una ley de potencia.

Palabras clave: Cocción de cerámicos, sinterizado, procesado, compactación térmica.

Stress distribution evolution in a ceramic body during firing. Part 1. Problem statement.

The study of stress profiles during ceramic firing is the main purpose of the present work. The characteristic problem for a variety of firing conditions, including hot pressing and traditional firing, is stated where the stress equilibrium is considered. In addition, several constitutive behaviors are taken into account. They are the Riedel model for polycrystals, the Scherer cell model for amorphous and the Kahn model for The Power Law Creep.
\end{abstract}

Key words: Ceramic firing, sintering, processing, thermal shrinkage.

\section{INTRODUCCIÓN}

La simulación del proceso de cocción de cerámicas es un instrumento que promete brindar beneficios significativos, entre otros, diseños más racionales y económicos en su elaboración. Este campo ya está siendo explorado por diversos investigadores, como son los casos de Cantavella [1], Riedel y col. [2-4], y Cedergren [5]. Sin embargo, en la generalidad de estos modelos se tienen restricciones por corresponder a intervalos isotérmicos o por ser bidimensionales o limitados en cuanto a geometría/composición.

La sinterización es el proceso físico, activado térmicamente, mediante el cual los granos de un compacto se sueldan entre sí. Este fenómeno ocurre a nivel mesoscópico, se basa en el transporte de material y da lugar a la compactación de los cerámicos durante la cocción [6]. La presencia de tensiones en este proceso puede ser fuente de deformaciones no deseadas y juega un papel importante en la sinterización asistida por presión.

En este estudio nos interesamos por la evolución de la distribución de tensiones en un cuerpo cerámico durante la cocción. Bordia y Scherer [7] plantean relaciones constitutivas macroscópicas para estudiar el sinterizado en presencia de tensiones. Consideramos el prensado en caliente de una muestra cilíndrica y el cocido tradicional de un laminado con tres capas de material, dos de ellas muy finas y una tercera más gruesa que asemeja una loza cerámica. Con esta información planteamos un modelo para el estudio de las tensiones.

\section{PLANTEAMIENTO DEL PROBLEMA}

La ecuación de equilibrio de tensiones es:

$$
\frac{\partial \sigma_{i j}}{\partial x_{j}}=0
$$

donde $\sigma_{i j}$ representa el tensor de tensiones y $x_{j}$ son las coordenadas. Estas coordenadas $\left(x_{1}, x_{2}, x_{3}\right)$ pueden ser transformadas al sistema cilíndrico $(r, \theta, z)$.

Las relaciones constitutivas para la sinterización en presencia de tensiones, pueden ser generalizadas:

$$
F\left(\dot{\varepsilon}_{i j}, \varepsilon_{i j}, \sigma_{i j}\right)=0
$$

siendo $F$ una relación de $\left(\dot{\varepsilon}_{i j}, \varepsilon_{i j}, \sigma_{i j}\right)$ donde

$$
\begin{aligned}
& \varepsilon_{i j}=\frac{1}{2}\left(\frac{\partial u_{i}}{\partial x_{j}}+\frac{\partial u_{j}}{\partial x_{i}}\right) \\
& \varepsilon_{i j}=\frac{d}{d t} \varepsilon_{i j}=\frac{1}{2}\left(\frac{\partial v_{i}}{\partial x_{j}}+\frac{\partial v_{j}}{\partial x_{i}}\right)
\end{aligned}
$$

$\varepsilon_{i j}$ es el tensor de tensiones en función de los desplazamientos $u_{i}$ y $\varepsilon_{i j}$ se conoce como velocidad de deformación en términos de las velocidades $v_{i}$. La forma específica que adopta la relación $F$ está determinada por el comportamiento constitutivo considerado en cada caso. En el presente trabajo se estudia el comportamiento lineal entre la tensión y la velocidad de deformación, el cual se denomina sinterización viscosa [7-10]. Para los casos del prensado en caliente también se tienen en 
cuenta los comportamientos constitutivos viscosos. Esto es valido para una presión aplicada relativamente baja la cual no introduce cambios considerables en la meso-estructura del cerámico. Para altas tensiones aplicadas el comportamiento viscosos no es una buena aproximación y como es sugerido por Ashby [11] se considera la ley de fluencia a la potencia. Khun y McMeeking [12] plantean un modelo que para el caso de prensado isostatico en caliente se reduce al modelo de Ashby. Este modelo puede ser interpretado como una de las formas de la ecuación (2).

Las condiciones de frontera son:

$$
\begin{gathered}
\left.\sigma_{i j}\right|_{B S}=\phi_{1}(B S) \\
u_{\left.i\right|_{B S}}=\left.\phi_{2}(B S) \quad o \quad v_{i}\right|_{B S}=\phi_{2}(B S)
\end{gathered}
$$

siendo $\phi_{1}(B S)$ y $\phi_{2}(B S)$ las funciones que describen el valor de las condiciones de contorno en la superficie de la frontera del cuerpo que sinteriza $B S$.

La correcta selección de las condiciones (4) y (5) más las ecuaciones (1) y (2) permiten plantear un modelo matemático cerrado y con solución única. A continuación se presentan los casos de interés del presente trabajo.

Estudiaremos la evolución de las tensiones para cuatro casos:

I. Se aplica una tensión uniaxial en la dirección $\mathrm{Z}$ de un cilindro cuyos bordes laterales están confinados (Fig. 1 (a)).

II. Similar al caso I, sólo que los bordes laterales del cilindro no están confinados (Fig. 1 (b)).

III. Se mueve un pistón en la dirección $Z$ a velocidad constante comprimiendo la muestra (Fig. 1 (b)). Los casos I, II y III son isotérmicos.

IV. La figura 2 muestra un cuerpo de forma rectangular con un espesor pequeño en comparación con las dimensiones de la base. Este cuerpo está compuesto de tres láminas superpuestas de materiales cerámicos como muestra la Fig. 2. A estas capas las llamamos, base, engobe y cuerpo y consideramos que el espesor de la base y el engobe es mucho menor que el del cuerpo. Por ejemplo una losa cerámica se puede describir así. Este cuerpo se somete a un programa de temperaturas contra tiempo y no se restringe ninguna deformación de la muestra. Luego, la diferencia entre el coeficiente de expansión térmica de los diferentes materiales y las diferencias en las dilataciones térmicas producto de la distribución de temperaturas en el cuerpo bajo un programa térmico dan lugar a la aparición de tensiones denominadas térmicas o composicionales.

\subsection{Casos I, II, y III: Prensado en caliente con comportamien- to lineal}

Para la sinterización viscosa la ecuación (2) tiene la forma:

$$
\sigma_{i j}=C_{i j k l} \dot{\varepsilon}_{k l}+\delta_{i j} \sigma_{s}
$$

donde $C_{i j k l}$ son los módulos viscosos, $\sigma_{s}$ es la tensión o potencial de sinterizado y $\delta_{i j}$ es la delta de Kronecker. Formalmente, se asume que la tensión de sinterizado describe la sinterización libre que ocurre en cuerpos sin ligaduras o restricciones.

Los cálculos se implementan en el software ANSYS 5.5 Multiphysics con el elemento axisimétrico PLANE42. Este elemento se desarrolla en dos dimensiones para problemas

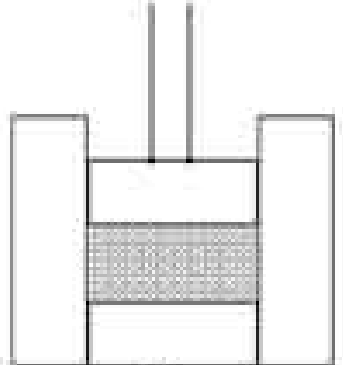

(a)

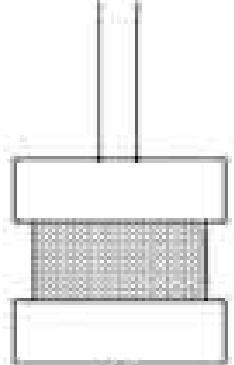

(b)
Fig. 1. Estaciones para el prensado en caliente. (a) caso I y (b) caso II, si una presión uniaxial, $P_{a}$, se aplica a través del pistón. Cuando una velocidad constante, $v$, se le impone al pistón la figura en (b) corresponde al caso III.

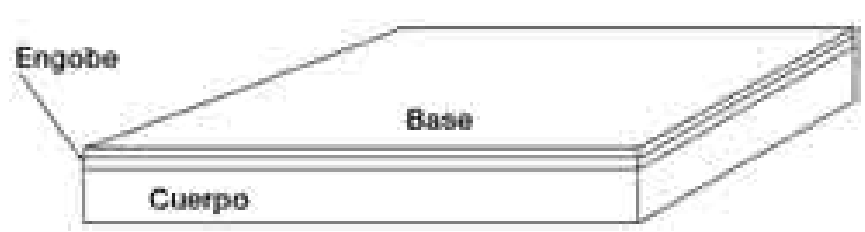

Fig. 2. Representación esquemática de una losa cerámica compuesta por el cuerpo, el engobe y la base.

estructurales, tiene forma de rectángulo y sus caras no tienen que ser paralelas, tiene un nodo en cada uno de sus cuatro vértices con dos grados de libertad (desplazamientos en 2D) por cada nodo. Este software se basa en el método de los elementos finitos, luego él tiene su forma para calcular el equilibrio de tensiones dictaminado por la ecuación (1). Siguiendo la metodología de trabajo de ANSYS nos es posible suministrar las condiciones de contorno (4) y (5). ANSYS tiene sus bibliotecas que contienen una gran cantidad de comportamientos constitutivos, PLANE42 considera que $\sigma_{s}=E_{i j k l} \varepsilon_{k l}$, donde $E_{i j k l}$ son los coeficientes elásticos. Considerando la analogía viscosa [7] tenemos que $E_{i j k l} \leftrightarrow C_{i j k l}$ y $\varepsilon_{k l} \leftrightarrow \varepsilon_{k l}$. El término $\delta_{i j} \sigma_{s}$ de la ecuación (6) no es tenido en cuenta por PLANE42. Entonces se hace necesario un ajuste del problema para su implementación en ANSYS.

Nos tomamos la libertad de definir la tensión ANSYS a través de la siguiente igualdad:

$$
\sigma_{i j}^{A}=C_{i j k l} \dot{\varepsilon}_{k l}
$$

Para el problema real tenemos las ecuaciones (1), (2), (4), y (5), luego, la Ec. (4) es escrita como:

$$
\left.\sigma_{i j}\right|_{B S}=\left(C_{i j k l} \varepsilon_{k l}+\delta_{i j} \sigma_{s}\right)_{B S}=\phi_{1}(B S)
$$

Re-acomodando esta ecuación tenemos que

$$
\left.C_{i j k l} \dot{\varepsilon}_{k l}\right|_{B S}=-\delta_{i j} \sigma_{s}+\phi_{1}(B S)
$$

La tensión de sinterizado es función de la temperatura y la densidad. Entonces, considerando que no hay gradientes de temperatura y densidad considerables en el compactado de polvos podemos afirmar que:

$$
\frac{\partial \sigma_{i j}}{\partial x_{j}}=\frac{\partial}{\partial x_{j}}\left(C_{i j k l} \dot{\varepsilon}_{k l}+\sigma_{s}\right)=\frac{\partial C_{i j k} \dot{\varepsilon}_{k l}}{\partial x_{j}}=\frac{\partial \sigma_{i j}^{A}}{\partial x_{j}}
$$


Finalmente, el problema en ANSYS para los casos I, II y III del presente trabajo y para los comportamientos viscosos descritos por el Modelo de Celdas y el Modelo de Riedel, presenta la siguiente forma:

$$
\begin{gathered}
\frac{\partial \sigma_{i j}^{A}}{\partial x_{j}}=0 \\
\left.\sigma_{i j}^{A}\right|_{B S}=-\delta_{i j} \sigma_{s}+\phi_{1}(B S) \\
\left.v_{i}\right|_{B S}=\phi_{2}(B S)
\end{gathered}
$$

La ecuación (12) nos sugiere que la tensión de sinterizado puede ser interpretada como una tensión hidrostática compresiva. Nuevamente resaltamos el hecho de que para los casos I, II y III los gradientes de temperatura y densidad son despreciables, entonces podemos considerar la muestra que sinteriza como homogénea. Si se le aplica a la muestra homogénea una tensión de extensión hidrostática y la misma es igual a la tensión de sinterizado $\left(\left.\sigma_{x}^{A}\right|_{B S}=\left.\sigma_{y}^{A}\right|_{B S}=\left.\sigma_{z}^{A}\right|_{B S}=-\sigma_{s}\right)$ las contracciones se detienen [7]. Esta es la misma interpretación que se puede deducir para el potencial de sinterizado en la ecuación (6) si planteamos el problema homogéneo $\left(\phi_{1}(B S) \equiv \phi_{2}(B S) \equiv 0\right)$. Entonces podemos concluir que el problema comprendido por las ecuaciones (1), (6), (4) y (5) es físicamente equivalentes al formado por las ecuaciones (11), (7) y (12). Si se usara otro software para la implementación numérica pude que el problema (11), (7) y (12) no sea el requerido, en cualquier caso se tendría que buscar un problema equivalente a (1), (6) (4) y (5).

A través del problema planteado se puede calcular el equilibrio de tensiones en un cuerpo que sinteriza con comportamiento viscoso, consideremos los materiales estudiados con propiedades isótropas, entonces la ecuación (6) queda de la forma:

$$
\begin{aligned}
& \sigma_{x}=\frac{E_{p}}{\left(1-2 v_{p}\right)\left(1+v_{p}\right)}\left[\left(1-v_{p}\right) \dot{\varepsilon}_{x}+v_{p}\left(\dot{\varepsilon}_{y}+\dot{\varepsilon}_{z}\right)\right]+\sigma_{s} \\
& \sigma_{y}=\frac{E_{p}}{\left(1-2 v_{p}\right)\left(1+v_{p}\right)}\left[\left(1-v_{p}\right) \dot{\varepsilon}_{y}+v_{p}\left(\dot{\varepsilon}_{z}+\dot{\varepsilon}_{z}\right)\right]+\sigma_{s} \\
& \sigma_{z}=\frac{E_{p}}{\left(1-2 v_{p}\right)\left(1+v_{p}\right)}\left[\left(1-v_{p}\right)_{z}+v_{p}\left(\dot{\varepsilon}_{x}+\dot{\varepsilon}_{y}\right)\right]+\sigma_{s} \\
& \sigma_{x y}=\frac{E_{p}}{1+v_{p}} \dot{\varepsilon}_{x y} \quad \sigma_{x z}=\frac{E_{p}}{1+v_{p}} \dot{\varepsilon}_{x z} \quad \sigma_{y z}=\frac{E_{p}}{1+v_{p}} \dot{\varepsilon}_{y z}
\end{aligned}
$$

donde $E_{p}$ es el módulo de viscosidad uniaxial, y $v_{p}$ es el coeficiente viscoso de Puasson. Entonces el detalle que queda es analizar la dependencia de las propiedades macroscópicas del sinterizado $\left(E_{p}, v_{p}\right.$ y $\left.\sigma_{s}\right)$ en función de la densidad relativa, $\rho, \mathrm{y}$ la temperatura, $T$.

Para el comportamiento viscoso consideramos dos casos, uno es el Modelo de Celdas de Scherer [8] que describe la sinterización de los materiales amorfos y el otro es el Modelo de Riedel $[9,10]$ para los materiales policristalinos.

Las formulas del el Modelo de Celdas [8] necesitadas en este trabajo son:

Potencial de sinterizado

$$
\sigma_{s}=-\left(\gamma n^{1 / 3}\right) \frac{\pi c_{1} c_{3} x^{5 / 3}}{\left(c_{1}-c_{2} x\right)^{2 / 3}}
$$

donde $x$ es un parámetro del Modelo de Celdas [8] y $n=1 / V_{\mathrm{s}}$ siendo $V_{s}$ el volumen de la parte sólida de la celda, $\gamma$ es la tensión superficial y $c_{1}=3 \pi, c_{2}=8 \sqrt{ } 2$ y $c_{1}=1$.

Los coeficientes de las relaciones constitutivas (13) tienen la siguiente forma:

$$
\begin{aligned}
& E_{p}=3 \eta c_{3} \pi x^{2} \\
& v_{p}=\left(\frac{3 c_{2}}{4 c_{1}}\right) x
\end{aligned}
$$

El Modelo de Celdas contiene la tensión superficial, $\gamma,(14)$ y la viscosidad , $\eta,(15)$. De estas dos la variable sobre la cual se tiene mayor control es la viscosidad debido a su intensa dependencia en función de la temperatura. Por ejemplo, para los silicatos, $\eta$ cambia un orden de magnitud por cada $20-40^{\circ} \mathrm{C}$, esta dependencia es aun más marcada en la vecindad de la temperatura de transición del vidrio. En cambio, la tensión superficial depende débilmente de la temperatura y de la composición, sus valores más bajos se reportan para el $\mathrm{P}_{2} \mathrm{O}_{5}$ siendo $\sim 0.06 \mathrm{~J} / \mathrm{m}^{2}$ y los más altos para la sílice siendo $\sim 0.3 \mathrm{~J} / \mathrm{m}^{2}$ [13]. Kingery y col. [6] reportan tensiones superficiales $\sim 1 \mathrm{~J} / \mathrm{m}^{2}$. Luego la dependencia térmica del sinterizado la estudiamos a través de la viscosidad. Si consideramos este proceso como un complejo térmicamente activado [14] que obedece la relación de Arrhenius, entonces tenemos:

$$
\eta=\eta_{0} \exp \left(\frac{\Delta U}{K T}\right)
$$

donde $\eta_{0}$ es la viscosidad de referencia, y $\Delta U$ es la energía de activación, $K$ es la constante de Boltzmann, y $T$ es el valor absoluto de la temperatura.

La densidad relativa como función del parámetro, $x$, se puede obtener a través de la relación:

$$
\rho=\left(\frac{c_{1}}{c_{0}}\right) x^{2}-\left(\frac{c_{2}}{c_{0}}\right) x^{3}
$$

siendo $c_{0}=1$. Nótese que la dependencia de la densidad relativa en función de $x$ indica que las propiedades del sinterizado son función de la densidad.

Las fórmulas del Modelo de Riedel $[9,10]$ usadas en el presente trabajo son:

La viscosidad del cuerpo, $K_{p}$, y la viscosidad de cizalladura, $G_{p}$, tienen la siguiente forma:

$$
\begin{aligned}
K_{p} & =\rho_{0}^{2 / 3} \rho^{1 / 3} Z \frac{K T c^{4}}{48 \Omega \delta D_{b} R} \\
G_{p} & =\rho_{0}^{2 / 3} \rho^{1 / 3} Z \frac{3 c^{2}}{20 R}\left[\frac{K T c^{2}}{12 \Omega \delta D_{b}}+\eta_{f}\right]
\end{aligned}
$$

luego, la viscosidad uniaxial, $E_{p}$, y el coeficiente de Poisson, $v_{p}$ pueden ser expresados como:

$$
\begin{aligned}
E_{p} & =\frac{9 K_{p} G_{p}}{\left(3 K_{p}+G_{p}\right)} \\
v_{p} & =\frac{3 K_{p}-2 G_{p}}{2\left(3 K_{p}+G_{p}\right)}
\end{aligned}
$$

donde $\rho_{0}$ es la densidad relativa inicial $\left(\left(\rho_{0}=0.7\right)\right), Z$ es el número de contactos de la partícula con sus vecinos $(Z=6), R$ es el radio inicial de las partículas, $\Omega$ es el volumen atómico, $\delta D_{b}$ es el coeficiente de difusión de la frontera por el ancho de la misma, $c$ es el área de contacto entre los granos, y $\eta_{f}$ es el parámetro de viscosidad para el deslizamiento entre los granos. Consideramos que esta viscosidad es función de la temperatura y los fenómenos difusionales en las fronteras de grano. Luego, como una aproximación podemos plantear que:

$$
\eta_{f}=B \frac{K T}{12 \Omega \delta D_{b}}
$$

siendo B un valor constante.

Los valores numéricos para los factores $c / R$ y $R \sigma_{s} / \gamma_{s}$ como función de la porosidad $f$ donde $\rho+f=1$ pueden ser encontrado en los trabajos de Riedel [9].

Para los materiales policristalinos (Modelo de Riedel), la dependencia de la temperatura está presente en el factor $(K T) /(\Omega \delta D b)$ de las ecuaciones (18) y (20). La difusión puede 
ser estudiada como un complejo térmicamente activado [6], entonces tenemos:

$$
D=D_{0} \exp \left(-\frac{\Delta U}{K T}\right)
$$

donde $D_{0}$ es el coeficiente de difusión de referencia. Consideremos que el ancho de la frontera de grano, $\delta$, no depende de forma considerable de la temperatura dentro del intervalo de la sinterización. En la presencia de expansiones térmicas podemos asumir que el volumen atómico, $\Omega$, depende de la temperatura de la siguiente manera [15]:

$$
\Omega=\text { Const } \times(K T)^{3}
$$

siendo Const un valor constante que depende de la composición del material.

Para completar el planteamiento del problema de las ecuaciones (11), (7) y (12), se deben dar las funciones $\varnothing_{1}$ y $\varnothing_{2}$ correspondientes a las condiciones de contorno (12). La tabla I muestra las relaciones asignadas a las mencionadas funciones dependiendo del caso que se trate I, II, o III.

Finalmente, se calculan las tensiones para cada caso según problema viscoso (policristales o amorfo) y haciendo uso de la velocidad de deformación se establecen los cambios en las dimensiones del cuerpo (Fig. 1) y se calcula la densidad del siguiente paso.

\subsection{Casos I, II, y III: Prensado en caliente con comportamien- to no lineal}

Para el prensado en caliente con comportamiento no lineal tomamos el modelo de Kuhn y McMeeking [12]. Para este modelo la velocidad de deformación se calcula a partir del estado de tensiones. Dicho modelo es planteado para el Estado I $(\rho<0.9)$. Los componentes del tensor de deformación de fluencia están dados por:

donde

$$
\dot{E}_{i j}=\dot{\varepsilon}\left(a \frac{3}{2} \frac{\sigma_{i j}+p \delta_{i j}}{q}-\frac{1}{3} b \delta_{i j}\right)
$$

$$
\begin{gathered}
\dot{\varepsilon}=\frac{2}{3} C_{c r}\left[|p|^{(n+1) / n}+\left(\frac{2 q}{3}\right)^{(n+1) / n}\right]^{n-1}\left[\frac{1}{2}|p|^{2 / n}+\left(\frac{2 q}{3}\right)^{2 / n}\right]^{1 / 2} \\
C_{c r}=A \frac{27 \pi \times 3^{1 / 2} \frac{\left(1-\rho_{0}\right)^{n-0.5}\left(\rho-\rho_{0}\right)^{0.5}}{\left[3 \rho^{2}\left(\rho-\rho_{0}\right)\right]^{n}}}{16}=\frac{\left(\frac{2 q}{3}\right)^{1 / n}}{\left[\frac{1}{2}|p|^{2 / n}+\left(\frac{2 q}{3}\right)^{2 / n}\right]^{1 / 2}} \\
b=\frac{\frac{3}{2}|p|^{1 / n} \operatorname{sign}(p)}{\left[\frac{1}{2}|p|^{2 / n}+\left(\frac{2 q}{3}\right)^{1 / 2}\right]^{1 / 2}}
\end{gathered}
$$

Las magnitudes $p$ y $q$ son la tensión hidrostática y de von Mises respectivamente, y están dadas por las fórmulas:

$$
\begin{aligned}
& p=-\frac{1}{3}\left(\sigma_{11}+\sigma_{22}+\sigma_{33}\right) \\
& q=\left(1 / 2\left[\left(\sigma_{1}-\sigma_{2}\right)^{2}+\left(\sigma_{2}-\sigma_{3}\right)^{2}+\left(\sigma_{3}-\sigma_{1}\right)^{2}\right]\right)^{1 / 2}
\end{aligned}
$$

TABLA I. COMPORTAMIENTOS CONSTITUTIVOS PARA LOS CASOS I, II, Y III Y SUS CORRESPONDIENTES CONDICIONES DE CONTORNOS SEGÚN LAS FUNCIONES $\phi_{1}(\mathrm{BS})$ Y $\phi_{2}(\mathrm{BS})$. SE DEDICAN TRES FILAS A LOS COMPORTAMIENTOS CONSTITUTIVOS Y TENEMOS LA COLUMNA I REFERENTE AL CASO I Y ASÍ SUCESIVAMENTE, LUEGO, POR EJEMPLO, RII SIGNIFICA QUE EL COMPORTAMIENTO CONSIDERADO ES EL DICTADO POR EL MODELO DE RIEDEL Y QUE NOS REFERIMOS AL CASO II. UNA INTERPRETACIÓN ANÁLOGA SE DEDUCE PARA LOS DEMÁS CASOS.

\begin{tabular}{|c|c|c|c|}
\hline & I & II & III \\
\hline $\begin{array}{c}\text { Ley de Fluencia a } \\
\text { la Potencia }\end{array}$ & PLCI & PLCII & PLCIII \\
\hline Riedel & RI & RII & RIII \\
\hline Scherer & SI & SII & SIII \\
\hline$\phi_{1}(z=h)$ & $\delta_{i j} P_{a}$ & $\delta_{i:} P_{r}$ & 0 \\
\hline$\phi_{2}(r=a, z=0, z=h)$ & $u_{r}=0, u_{z}=0, n o B C$ & $n o B C, u_{z}=0, n o B C$ & no $B C, v_{z}=0, u_{z}=-v \times t$ \\
\hline
\end{tabular}

siendo $\sigma_{1}, \sigma_{2}$ y $\sigma_{3}$ las componentes principales del tensor de tensiones

$$
A=A^{*} \exp \left(-\frac{\Delta U}{k T}\right)
$$

donde $\Delta U$ es la energía de activación y $\mathrm{A}^{*}$ es el valor de referenica del coeficiente térmico A de la ecuación (24)".

La ecuación (23) es una de las formas que puede tomar la relación (2), pero para este caso no resolvemos el sistema formado por las ecuaciones (1), (23), (4) y (5). Como proponen Bhattacharya y col. [12] se plantea un problema elástico para el cálculo de las mencionadas tensiones. Entonces el problema que se resuelve es el formado por las ecuaciones (1), (2), (4) y (5), donde (2) toma la forma de la Ley de Hooke para los materiales isótropos. De esta forma se calcula el estado de tensiones que se importa en la ecuación (23).

Entonces, consideramos la dependencia del Modulo de Young respecto a la porosidad con la fórmula [16]:

$$
E=E_{o}\left(1-\frac{f}{f_{0}}\right)^{\alpha}
$$

donde $f_{0}$ y $\alpha$ son parámetros de correlación empírica.

Las condiciones de contorno (Ec. (4) y (5)) del mencionado problema elástico se resumen en la Tabla I. En los códigos ANSYS se incluye una subroutina que implementa el modelo de Kuhn.

Entonces el procedimiento consiste en:

1. Se calculan las tensiones según problema elástico

2. Se calculan las deformaciones acorde al Modelo de Kuhn.

3. Se re-establecen las dimensiones del cuerpo (Fig. 1) y se calcula la densidad del paso siguiente.

\subsection{Caso IV: Cocción tradicional}

Para el caso IV como muestra la figura 2 y como fue anteriormente explicado, estudiamos un cuerpo que está compuesto por tres materiales cerámicos diferentes. Además, este cuerpo está sometido a un programa térmico que provoca la presencia de una distribución de temperaturas. Las propiedades de la sinterización dependen de la composición química 
y la temperatura, entre otros parámetros como por ejemplo la densidad. Entonces, este cuerpo va a experimentar diferentes velocidades de densificación en sus diferentes elementos diferenciales de volumen, lo cual provoca la aparición inevitable de tensiones composicionales causas de deformaciones no deseadas, por ejemplo encorvamiento de la pieza.

Para el cálculo de las tensiones composicionales en este caso se sigue una secuencia de problemas.

1. Se resuelve un problema térmico con la ayuda de SOLID70, el cual es tridimensional y tiene forma de prisma deformado con sus caras no necesariamente paralelas. Tiene 8 nodos, uno en cada vértice y su único grado de libertad es la temperatura. Las propiedades del material requeridas son el coeficiente de conductividad térmica, $K_{T}$ y la capacidad calorífica, $c_{p}$.

2. Se importa la distribución de temperaturas obtenida a través del problema térmico en un problema termo-elástico con SOLID45 donde se considera

$$
\sigma_{i j}=E_{i j k l}\left(\varepsilon_{k l}+\delta_{i j} \delta_{k l} \alpha_{T} \Delta T\right)
$$

siendo $\alpha_{T}$ el coeficiente de dilatación térmica y $\Delta \mathrm{T}=T-T_{\text {ref }}$ donde $T$ es la temperatura y $T_{r e f}$ es la temperatura de referencia. Este elemento es el equivalente estructural de SOLID70, su diferencia más notable es que por cada nodo tiene tres grados de libertad, estos son los desplazamientos en las tres direcciones independientes del espacio.

La dependencia del módulo de Young en función de la porosidad se tiene en cuenta a través de la fórmula (27), la dependencia térmica se toma como lineal con pendiente negativa, de esta forma consideramos de forma aproximada la disminución del módulo elástico con el aumento de la temperatura. Como se muestra en la parte 2, el coeficiente de dilatación térmica respecto de la temperatura se toma de diferentes formas en diferentes experimentos.

3. La distribución de tensiones composicionales del problema termo-elástico se importa en un problema viscoso con el comportamiento constitutivo según (13), (15), (16) y (17), es decir, sólo tenemos en cuenta el modelo de Scherer. Este problema es el que describe la distribución de tensiones durante la cocción.

Este ciclo se repite por la cantidad de pasos que se considere que pasa la pieza cerámica durante el tratamiento térmico.

\section{CONCLUSIONES}

Se ha planteado un problema para simular la evolución de las tensiones durante la cocción bajo las condiciones de prensado en caliente y cocción tradicional.

El presente problema considera la dependencia de las propiedades que gobiernan la cocción con la temperatura y de la densidad, además se pueden tener en cuenta variados perfiles térmicos de cocción. El presente modelo considera comportamientos viscosos y no lineales y se pueden considerar compuestos, como por ejemplo compuestos laminares. Este nivel de integración representa una contribución modesta difícil de encontrar en la literatura.

Se plantea que deben de existir tensiones responsables de las deformaciones no deseadas o no esperadas durante la cocción, a estas tensiones se les llama composicionales y actúan en el caso de la cocción tradicional. Para el caso de la sinterización viscosa estas tensiones tienen su origen en los gradientes de temperatura y densidad que provocan variaciones de la viscosidad en el volumen del cuerpo. Para el caso de un material compuesto al factor que se acaba de mencionar se debe agregar las diferencias entre las dilataciones térmicas de los diferentes materiales.

Para el caso del prensado en caliente la confinación a la que se somete el cuerpo provoca distribuciones de tensiones. Además se tiene que para el caso de una razón de deformación impuesta a través de un pistón el cuerpo reacciona con tensión.

En fin, la presencia de tensiones durante la cocción de cerámicos es un aspecto a ser tenido en cuenta con vistas a estudiar diversos fenómenos relacionados a la cocción como la densificación, las deformaciones y el desarrollo mesoestructural entre otros.

\section{AGRADECIMIENTOS}

Este trabajo es resultado de las investigaciones apoyadas por el Proyecto CONACYT 31234-U.

De igual manera agradecemos a Internacional de Cerámica S.A. de C.V. (Interceramic) por su apoyo para el desarrollo del presente trabajo.

\section{BIBLIOGRAFÍA}

1. Vicente Cantavella Soler. Simulación de la deformación de baldosas cerámicas durante la cocción. Tesis doctoral Castellón, 1998.

2. D.-Z. Sun \& H. Riedel. Prediction of Shape distortions of hard metal parts by numerical simulation of pressing and sintering. Simulation of Materials Processing: Theory, methods and Applications, Shen \& Dawson (eds) 1995 Balkema, Rotterdam, ISBN 9054105534.

3. T. Kraft, H. Riedel, O. Rosenfelder and P. Stingl. Computational optimization of Parts Produced from Ceramic Powders, in: Microstructures, Mechanical Properties and Processes, Y. Bréchet (ed.), EUROMAT 1999, Vol. 3, WileyVCH, Weinheim, Germany (2000) 337-342.

4. T. Kraft, O. Coube and H. Riedel, Numerical Simulations of Pressing and Sintering in the Ceramic and hard Metal Industry, in: Recent Developments in Computer Modelling of Powder Metallurgy Processes, NATO Advanced research Workshop, Series III: Computer and Systems Science - Vol. 176, A. Zavaliangos, A. Laptev (eds.), IOS Press, Amsterdam, Netherlands (2001) $181-190$.

5. J Cedergren, NJ Sorensen, A. Bergmark. Three-dimensional analysis of compaction of metal powder. Mechanics of Materials 24 (2002) 43-59.

6. W.D. Kingery. H.K. Bowen. y D.R. Uhlmann. Introduction to Ceramics. Jhon Wiley \& Sons. 1975.

7. R. K. Bordia and G. W. Scherer. On constrained Sintering- I. Constitutive Model for a sintering body. (pp. 2393-2397) II. Comparison of Constitutive Models. (pp. 2399-2409) III. Rigid Inclusions. Acta Metall. Vol. 36, No. 9. 1988.

8. G. W. Scherer, "Cell Models for Viscous Sintering," J. Am. Ceram. Soc., 74 [7] 1523-31 (1991)

9. J. Svoboda, H. Riedel and H. Zipse, “Equilibrium Pore Surfaces, Sintering Stresses and Constitutive Equations for the Intermediate and Late Stage of Sintering-I. Computation of Equilibrium Surfaces," Acta metall. mater., 42 [2] 435-443 (1994)

10. H. Riedel, H. Zipse and J. Svoboda, "Equilibrium Pore Surfaces, Sintering Stresses and Constitutive Equations for the Intermediate and Late Stage of Sintering-II. Diffusional Densification and Creep," Acta metall. mater., 42 [2] 445-452 (1994).

11. A. S. Helle, K. E. Easterling, and M. F. Ashby, "Hot-isostatic pressing diagrams: new developments," Acta Metall., 33 [12], 2163-74 (1985).

12. S. Bhattacharya, K. Jakus, I. Grosse, "Modelling pressure-assisted densification by power-law creep," J. Mat. Sci. 32 6183-6189 (1997).

13. G.W. Scherer. Viscvous sintering of Inorganic Gels. Capter 4. Surface and Colloidal Science, Vol 14. Edited by Egon Matijevic. Plenum Publishing Corporation, 1987

14. Randall M. German. Sintering Theory and Practice. Jhon Wiley \& Sons, Inc. 1996.

15. Charles Kittel. Introduction to Solid State Physics. Seventh Edition. Jhon Wiley \& Sons. 1996.

16. A.P. Roberts and E.J. Garboczi. Elastic Properties of model Porous Ceramics. J. Am. Ceram. Soc., 83 [12] 3041-48 (2000).

Recibido: 03.03 .03

Aceptado: 12.09 .03 